\title{
Composition and Decomposition of Internal Models in Motor Learning under Altered Kinematic and Dynamic Environments
}

\author{
J. Randall Flanagan, ${ }^{1}$ Eri Nakano, ${ }^{2}$ Hiroshi Imamizu, ${ }^{3}$ Rieko Osu, ${ }^{3}$ Toshinori Yoshioka, ${ }^{3}$ and Mitsuo Kawato ${ }^{2,3}$ \\ ${ }^{1}$ Department of Psychology, Queen's University, Kingston, Ontario K7L 3N6, Canada, ${ }^{2}$ ATR International, Kyoto 619- \\ 0288, Japan, and ${ }^{3}$ Kawato Dynamic Brain Project, Exploratory Research for Advanced Technology, Japan Science and \\ Technology Corporation, Kyoto 619-0288, Japan
}

The learning process of reaching movements was examined under novel environments whose kinematic and dynamic properties were altered. We used a kinematic transformation (visuomotor rotation), a dynamic transformation (viscous curl field), and a combination of these transformations. When the subjects learned the combined transformation, reaching errors were smaller if the subject first learned the separate kinematic and dynamic transformations. Reaching errors under the kinematic (but not the dynamic) transformation were smaller if subjects first learned the combined transformation. These results suggest that the brain learns multiple internal models to compensate for each transformation and has some ability to combine and decompose these internal models as called for by the occasion.

Key words: motor learning; reaching; internal model; visuomotor transformation; force fields; arm movement
Most purposeful actions, including tool use, involve significant interactions with the environment. The motor commands required to perform such actions depend not only on the kinematics (Lacquaniti et al., 1995) and dynamics (Kalaska et al., 1989) of the musculoskeletal system but also on the kinematics and dynamics of manipulated tools and the environment. The ability of humans to adapt to a range of environments and to easily switch between familiar environments indicates that the CNS learns and maintains internal models of the kinematics and dynamics of different environments.

A fundamental question related to internal models concerns their granularity. Here the issue is whether CNS uses a small number of global internal models or whether it maintains a large number of internal models or modules for different contexts. For a global internal model to adapt to different sensorimotor contexts, it must learn the properties of tools and environments whenever they are altered-even if these properties have been learned previously. On the other hand, if the CNS uses multiple internal models, each model could learn the properties of a particular environment or tool, and there would be less relearning involved. Moreover, initial learning of tools and environments may be facilitated by combining stored modules (Ghahramani and Wolpert, 1997). The recently proposed multiple internal model hypothesis (Kawato and Wolpert, 1998; Wolpert and Kawato, 1998; Wolpert et al., 1998) argues for motor control and learning based on such a modular strategy. This model assumes that separate internal models are learned for different environments and also permits mixtures of internal models to cope with a single environment or task.

Several lines of evidence support the multiple internal model

\footnotetext{
Received July 8, 1999; revised Aug. 18, 1999; accepted Aug. 20, 1999.

This work was supported by the Human Frontier Science Program, the Japan Science and Technology Agency, and the Natural Sciences and Engineering Research Council of Canada.

Correspondence should be addressed to J. Randall Flanagan, Department of Psychology, Queen's University, Kingston, Ontario, Canada K7L 3N6. E-mail: flanagan@psyc.queensu.ca.

Copyright (C) 1999 Society for Neuroscience $\quad 0270-6474 / 99 / 190001-\bullet \$ 05.00 / 0$
}

hypothesis. Brashers-Krug et al. (1996) and Shadmehr and Brashers-Krug (1997) examined adaptations to unusual force fields in reaching movements. They have shown that subjects are able to learn internal models of multiple force fields, and that these models can be successfully recalled, with little decrement in performance, for up to 5 months and probably longer. Previous work on learning in reaching tasks has demonstrated that humans are able to adapt to a wide range of visuomotor (MacGonigle and Flook, 1978; Welch, 1986; Welch et al., 1993; Flanagan and Rao, 1995; Imamizu and Shimojo, 1995; Imamizu et al., 1995; Ghahramani and Wolpert, 1997) and dynamic (Shadmehr and MussaIvaldi, 1994; Brashers-Krug et al., 1996; Conditt et al., 1997; Flanagan and Wing, 1997; Sheidt et al., 1997) transformations. However, little is known about how the CNS deals with novel environments in which the kinematic and dynamic properties are altered simultaneously.

The first goal of the current study was to test the hypothesis that the CNS can effectively combine previously learned internal models when encountering a novel environment in which the previously learned sensorimotor transformations are simultaneously presented (combined transformation). The second goal was to test the related hypothesis that the CNS can decompose the previously learned combined transformation when encountering the separate (and novel) component transformations. To evaluate these hypotheses, we used visuomotor ( $\pm 60^{\circ}$ rotation)

This article is published in The Journal of Neuroscience, Rapid Communications Section, which publishes brief, peerreviewed papers online, not in print. Rapid Communications are posted online approximately one month earlier than they would appear if printed. They are listed in the Table of Contents of the next open issue of JNeurosci. Cite this article as: JNeurosci, 1999, 19:RC34 (1-5). The publication date is the date of posting online at www.jneurosci.org.

http://www.jneurosci.org/cgi/content/full/3586 
and dynamic (viscous curl fields of opposite sign) transformations either separately or in combination. In the composition experiment, subjects first learned separate visuomotor and dynamic transformations and then the combined transformation. In the decomposition experiment, the same subjects first learned the combined transformation and then the separate transformations. Transformations of opposite sign were used in the two experiments to guard against transfer of learning, and the two experiments were separated by at least 1 week to guard against interference effects (Shadmehr and Brashers-Krug, 1997).

We hypothesized that performance under the combined transformation would be facilitated by previous learning of the separate transformations. This would indicate that subjects are able to effectively combine the previously learned visuomotor and dynamic internal models. We also hypothesized that performance under the separate transformations would be facilitated by previous learning of the combined transformation. This would suggest that the CNS is able to decompose the combined transformation (or an internal model of the combined transformation) into separate internal models.

\section{MATERIALS AND METHODS}

Subjects. Six males and two females, 21-35 years old, participated in these experiments after giving informed consent. None of the subjects reported sensorimotor or neurological problems, and all had correct-fornormal vision. All of the subjects were naive with respect to the hypotheses under study, and none had previously experienced the sensorimotor transformations examined.

Apparatus. Subjects sat on a chair, held the tip of a force-reflecting manipulandum (Gomi and Kawato, 1996) with the right hand and executed reaching movements in the horizontal plane to visually presented targets. The arm was supported by either a strap from the ceiling or a brace fixed to the manipulandum. The current hand position (a cursor 0.4 $\mathrm{cm}$ in diameter) measured by the manipulandum and the target circle (1 $\mathrm{cm}$ in diameter) were indicated on a large cathode ray tube (CRT) screen located $1.6 \mathrm{~m}$ in front of the subject. The scales of the CRT coordinates and hand coordinates were the same. The position of the hand and the forces applied by the hand to the manipulandum were sampled at $500 \mathrm{~Hz}$. The subject performed the task only by looking at the CRT screen; a board occluded vision of the arm.

Procedure. Subjects were asked to move the cursor quickly and accurately to a series of targets that appeared in succession on the screen. Each target served as the start position for the next movement. Targets were randomly positioned within the work space $(14 \mathrm{~cm}$ in radius) but were constrained to be $10 \mathrm{~cm}$ from the start position. Each new target was presented for $600 \mathrm{msec}$ and then extinguished. After a short delay, the next target appeared. Targets were presented in sets of 10. At the start of each set the subject positioned the cursor in the center of the work space.

Each subject completed the composition and decomposition experiments at least 1 week apart with the order counterbalanced across subjects. Both experiments consisted of four transformation conditions: normal, visuomotor, dynamic, and combined (visuomotor and dynamic). The normal condition was included to familiarize subjects with the manipulandum.

In both experiments, subjects first completed 30 sets of 10 trials in the normal condition. In the composition experiment they then completed 50 sets of trials in the visuomotor and dynamic conditions (counterbalanced across subjects) followed by 50 sets of trials in the combined condition. In the decomposition experiment, subjects completed 50 sets of trials in the combined condition followed by 50 sets in the visuomotor and dynamic conditions (again counterbalanced across subjects). The subjects took brief rests between transformation conditions.

The normal, rotational, and viscous transformations are coded $\mathrm{N}, \mathrm{R}$, and $\mathrm{B}$, respectively and the combined transformation is coded $\mathrm{R}+\mathrm{B}$. Superscripts are used to indicate the perturbation direction (see below). To guard against practice effects across experiments (i.e., weeks), the directions of the transformations were reversed for each subject. $\mathrm{R}^{\prime}$ and $\mathrm{B}^{\prime}$ denote transformations with signs opposite $\mathrm{R}$ and $\mathrm{B}$ respectively. Previous work on adaptation to viscous force fields (Shadmehr and Brashers-Krug, 1997) and visuomotor rotations (E. Nakano, unpublished data) has revealed that there are no positive or negative transfer effects when the direction of the perturbation is reversed and the perturbations are delivered $>24 \mathrm{hr}$ apart. We assumed that $\mathrm{R}$ and $\mathrm{R}^{\prime}, \mathrm{B}$ and $\mathrm{B}^{\prime}$, and $\mathrm{R}+\mathrm{B}$ and $\mathrm{R}^{\prime}+\mathrm{B}^{\prime}$ were equivalent in terms of difficulty.

Transformation rules. In the rotational transformation, the subjects controlled the position of the cursor $(x, y)$ which corresponded to the position of the actual hand $(p, q)$ rotated about the center of the work space:

$$
\left(\begin{array}{l}
x \\
y
\end{array}\right)=\mathrm{R}\left(\begin{array}{l}
p \\
q
\end{array}\right)=\left(\begin{array}{cc}
\cos \theta & -\sin \theta \\
\sin \theta & \cos \theta
\end{array}\right)\left(\begin{array}{l}
p \\
q
\end{array}\right) .
$$

Two rotation matrices were used: $\mathrm{R}^{+}$where $\theta=60^{\circ}$ and $\mathrm{R}^{-}$where $\theta=-60^{\circ}$.

For the viscous transformation, we used the same type of viscous curl force fields used by Shadmehr and Mussa-Ivaldi (1994). The manipulandum produced forces $\left(f_{x}, f_{y}\right)$ on the subject's hand that were proportion to the velocity of the hand $(\dot{p}, \dot{p})$ :

$$
\left(\begin{array}{c}
f_{x} \\
f_{y}
\end{array}\right)=\mathrm{B}\left(\begin{array}{c}
\dot{p} \\
\dot{q}
\end{array}\right)=\left(\begin{array}{cc}
\alpha & \chi \\
\chi & -\alpha
\end{array}\right)\left(\begin{array}{c}
\dot{p} \\
\dot{q}
\end{array}\right)
$$

Two viscosity matrices we used: $\mathrm{B}^{+}$where $\alpha=13 \mathrm{~N} \cdot \mathrm{m}^{-1} \cdot \mathrm{sec}^{-1}$ and $\mathrm{B}^{-}$where $\alpha=-13 \mathrm{~N} \cdot \mathrm{m}^{-1} \cdot \mathrm{sec}^{-1} \cdot \chi$ was always $-13 \mathrm{~N} \cdot \mathrm{m}^{-1} \cdot \mathrm{sec}^{-1}$.

Data analysis. The position data were digitally filtered using a fourthorder low-pass Butterworth filter with a cutoff frequency of $20 \mathrm{~Hz}$. Velocities were computed with a three-point local polynomial approximation. The start and end of each movement were defined as the points at which the curvature of the two-dimensional path of the hand first exceeded and then subsequently dropped below $3 \mathrm{~mm}^{-1}$, respectively (Imamizu et al., 1995). Defined in this way, the end of the movement occurs before small corrective movements often observed near the target.

To quantify trajectory learning, we computed two measures of performance. The target error was defined as the distance between the target and end positions. This error has previously been used to study adaptation under rotational transformations (Imamizu et al., 1995). The path distance was defined as the length or distance traveled by the hand. Shadmehr and Mussa-Ivaldi (1994) demonstrated that during adaptation to viscous force fields, hand paths become less and less curved and eventually become approximately straight. Thus, the path distance decreases with learning. The target errors and path distances were averaged across the 10 trials within each set. Thus, for each measure, we obtained 30 values in the normal condition and 50 values in the rotational, viscous, and combined conditions. In this paper, we focus on the first 30 sets of trials in each condition.

Repeated measures ANOVAs were used to assess various experimental effects on the two trajectory measures. A significance level of 5\% was considered statistically reliable.

\section{RESULTS}

We first provide a brief qualitative description of the results using single-trial data from a single subject and then present the results, in quantitative form, using data averaged across subjects.

\section{Single-trial data}

\section{Hand paths}

Examples of hand paths in early (gray traces) and later (black traces) stages of learning are shown in Figure 1 for both the composition experiment and the decomposition experiment (data from subject R.B.). Under the normal transformation, the hand paths were almost straight, and the target errors were small both in early and late trial sets. In the early stage of learning in the composition experiment, large directional errors in the hand path were observed under the rotational transformation $\left(\mathrm{R}^{+}\right)$, and curved and misdirected hand paths were also seen under viscous transformation $\left(\mathrm{B}^{-}\right)$. Under the combined transformation $\left(\mathrm{R}^{+}+\right.$ $\mathrm{B}^{-}$), deviations in the hand paths early in learning were generally small in comparison with the deviations observed in early learning under the previously encountered rotational and viscous transformations. In the early stage of learning in the decomposition experiment, large deviations in the hand paths were observed under the combined transformation $\left(\mathrm{R}^{-}+\mathrm{B}^{+}\right)$. However, the 


\section{Composition}

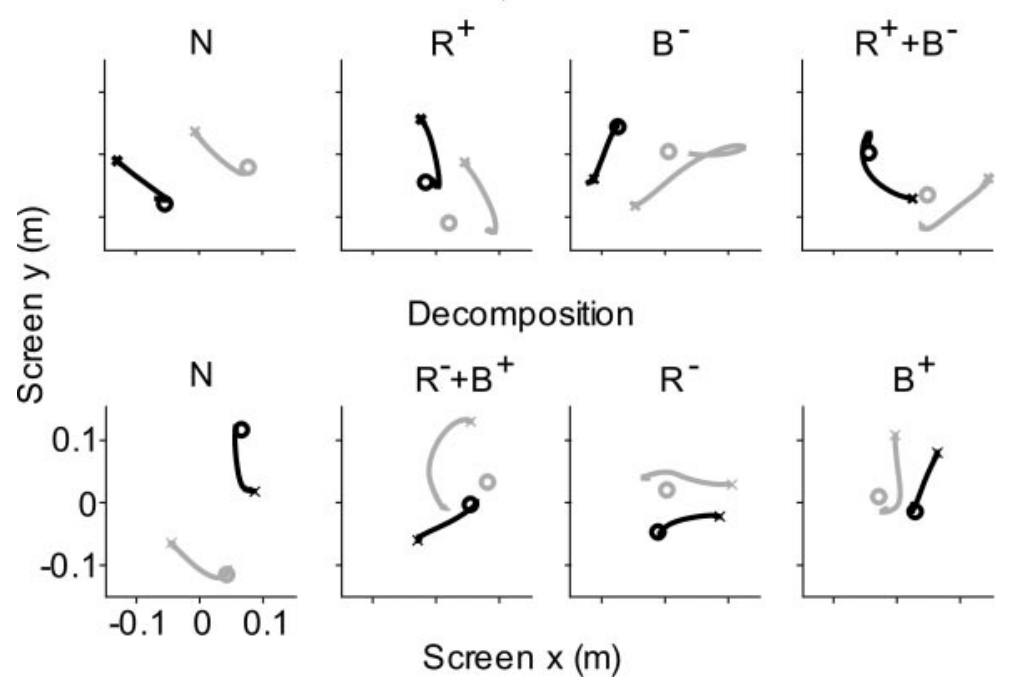

Figure 1. Single hand paths measured under each transformation in the composition (top) and decomposition (bottom) experiments. Hand paths are shown for trials performed in the early stage of learning (1st set; gray traces) and in the late stage of learning (30th set; black traces). $N, R, B$, and $R+B$ denote the normal, rotational, viscous, and combined transformations with superscripts indicating the direction of the transformation. The initial $(x)$ hand position and target position $(o)$ are indicated for each trial. Data are from subject R.B.
Comp. Dec.
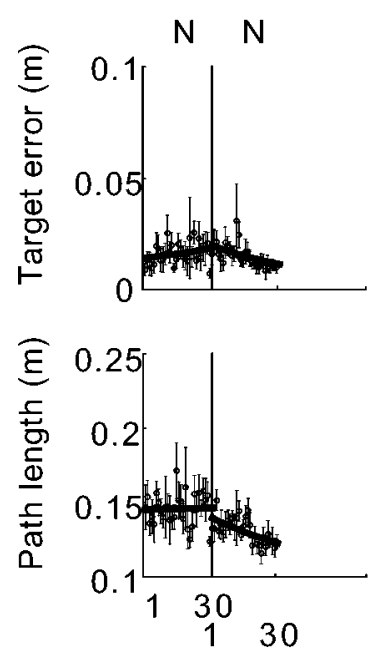

Comp. Dec.
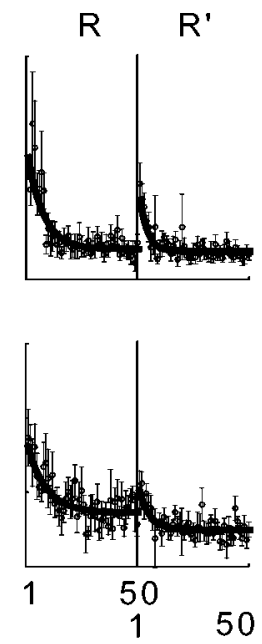

Comp. Dec.
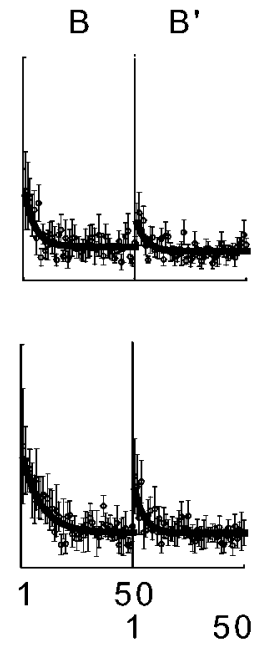

Trial Set deviations under the rotational $\left(\mathrm{R}^{-}\right)$and viscous $\left(\mathrm{B}^{+}\right)$transformations, encountered after learning the combined transformation, were relatively small. Under all transformations, nearly straight hand paths were eventually observed after adaptation.

\section{Learning curves}

Figure 2 shows, for subject R.B., target errors and path distances as a function of trial set for each transformation condition. The left and right sides of each panel show the errors or distances obtained without and with previous learning of the complementary transformation(s), respectively. Thus, this subject experienced $\mathrm{R}$ and $\mathrm{B}$ before $\mathrm{R}+\mathrm{B}$ (composition experiment) but experienced $\mathrm{R}^{\prime}$ and $\mathrm{B}^{\prime}$ after $\mathrm{R}^{\prime}+\mathrm{B}^{\prime}$ (decomposition experiment). For the normal transformation, the left and right sides of the panels show data obtained in the composition and decomposition experiments, respectively. For illustrative purposes, exponentials of the form $k_{0}+k_{1}{ }^{*} \exp \left(-k_{2}{ }^{*} n\right)$, where $n$ denotes the set number and $k_{\mathrm{i}}$ denotes a constant coefficient, were fit to each set of data.

For this subject, performance under the separate rotational $\left(\mathrm{R}^{\prime}\right)$ and viscous $\left(\mathrm{B}^{\prime}\right)$ transformations was clearly facilitated by previous learning of the combined $\left(\mathrm{R}^{\prime}+\mathrm{B}^{\prime}\right)$ transformation. Sim-
Dec. Comp.
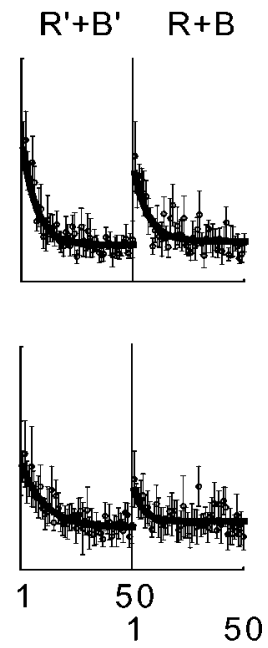

Figure 2. Changes in target error and path distance across trial sets under each transformation for subject R.B. For the normal $(N)$, rotational $(R)$, and viscous $(B)$ transformations, the left sides show data from the composition experiment (Comp.) in which $\mathrm{R}$ and $\mathrm{B}$ were tested before the combined transformation $(R+B)$. The right sides show data from the decomposition (Dec.) experiment in which $\mathrm{R}^{\prime}$ and $\mathrm{B}^{\prime}$ were tested after learning $\mathrm{R}^{\prime}+\mathrm{B}^{\prime}$. For the combined transformation, the left and right sides show performance without and with previous learning of the separate transformations, respectively. Exponential functions have been fit to each set of data (see Results for details).

ilarly, performance under the combined transformation $(\mathrm{R}+\mathrm{B})$ was facilitated by previous learning of the two separate transformations ( $\mathrm{R}$ and $\mathrm{B})$. However, transfer of learning was not perfect. Whereas the initial target errors and path distances were smaller after previous learning of the complementary transformation(s), they also tended to be greater than the errors and distances observed at the end of the previous learning.

\section{Averaged data}

To characterize performance under each transformation condition, we first computed subject averages, for both target error and path distance, over the first 10 sets of trials and over sets 21-30. Thus, we characterized the initial performance under each transformation as well as later performance. We then computed means and SDs based on the subject averages. Figure 3 shows, for each transformation, the mean target errors and path distances during both early learning (circles) and later performance (squares). The errors observed with ( filled symbols) and without (open symbols) previous learning of the complementary transformation(s) are joined by lines. The stars indicate a reliable difference $(p<0.05)$ 


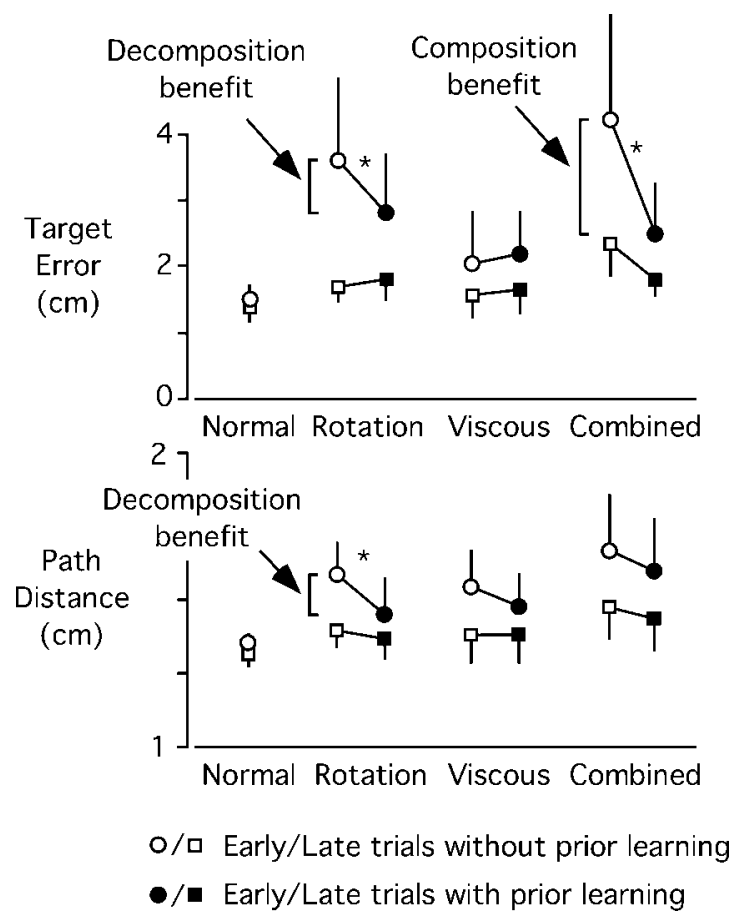

Figure 3. Mean target errors (top) and path distances (bottom) during initial learning (circles) and later performance (squares) under each of the four transformation conditions. For the rotational and viscous transformations, the closed and open symbols represent performance with and without prior learning of the combined transformation. For the combined transformation, the filled and open symbols represent performance with and without prior learning of the separate rotational and viscous transformations. Error bars represent SDs. Stars indicate a statistically significant difference between pairs of transformations with and without prior learning.

between pairs of transformations (with and without previous learning), and the error bars represent SDs.

Figure 3 reveals that hand trajectories were clearly altered during initial learning by the visuomotor, dynamic, and combined transformations. Without previous learning of the complementary transformations (open circles), both targets errors $\left(F_{(1,7)}=\right.$ $39.1 ; p<0.001)$ and path distances $\left(F_{(1,7)}=60.2 ; p<0.001\right)$ were reliably greater under the rotational, viscous, and combined transformations (grouped together) than under the normal transformation. Even with previous learning of the complementary transformations ( filled circles), initial target errors $\left(F_{(1,7)}=28.9\right.$; $p=0.001)$ and path distances $\left(F_{(1,7)}=16.4 ; p=0.005\right)$ were reliably greater under the rotational, viscous, and combined transformations than under the normal transformation. Overall, initial performance under the non-normal transformations was better with previous learning (open circles) than without (filled circles $)$ both in terms of target error $\left(F_{(1,7)}=17.4 ; p=0.004\right)$ and path distance $\left(F_{(1,7)}=17.0 ; p=0.004\right)$.

\section{Transfer of learning in composition}

To assess the composition hypothesis, we compared initial performance under the combined transformation with and without previous learning of the separate transformations. If previous learning of the separate transformations facilitates performance under the combined condition, then the initial performance should be better than that observed without previous learning. Repeated measures ANOVA revealed that the mean target error was significantly smaller $\left(F_{(1,7)}=9.05 ; p=0.02\right)$ with previous learning than without (see Fig. 3, Composition benefit). However, the mean path distances with and without previous learning were not reliably different $\left(F_{(1,7)}=0.81 ; p=0.40\right)$. These results indicate that performance in the combined transformation condition was facilitated by previous learning of the separate visuomotor and viscous transformations. This improvement in performance was reliably observed in the target error.

\section{Transfer of learning in decomposition}

To test the decomposition hypothesis, we compared initial performance under the separate transformations with and without previous learning of the combined transformation. We will first consider the rotation transformation. The mean path distance was significantly smaller with previous learning than without $\left(F_{(1,7)}=\right.$ 8.67; $p=0.02$ ). Similarly, the mean target error with previous learning was reliably smaller than without previous learning $\left(F_{(1,7)}=6.24 ; p=0.04\right)$. Thus, for the visuomotor transformation condition, the results clearly indicate that previous learning of the combined transformation facilitated performance (see Fig. 3, Decomposition benefit). In contrast, such transfer of learning was not observed under the viscous transformation. Reliable effects of previous learning of the combined transformation were not observed on mean initial path distance $\left(F_{(1,7)}=1.22 ; p=0.31\right)$ or mean initial target error $\left(F_{(1,7)}=0.15 ; p=0.71\right)$.

\section{Order effects}

We used viscous force fields and visuomotor rotations of opposite signs to guard against practice effects across experiments (i.e., weeks). Nevertheless, to assess possible effects of practice, we compared the performance between the second and third transformations in the composition experiment and between the third and fourth transformations in the decomposition experiment. Thus, only the rotation and viscous transformations were considered. In both experiments, half the subjects received the rotation transformation followed by the viscous transformation, and the other half received the transformations in the opposite order. Therefore, we were able to assess the effects of order while counterbalancing across the two types of transformation. Repeated measures ANOVAs were used to assess the effects of order on target error and path distance in both experiments. Thus four separate ANOVAs were performed, two for each experiment. No reliable effects of order were observed $(p>0.20$ in all cases). Thus, performance under the second transformation (third or fourth) was not reliably different from performance under the first (second or third).

\section{DISCUSSION}

The present study tested two general hypotheses concerning internal models of sensorimotor transformations. The composition hypothesis holds that the CNS can effectively combine internal models of two previously learned sensorimotor transformations when dealing with a novel environment in which both transformations are present. The decomposition hypothesis holds that, when encountering a complex environment featuring more than one sensorimotor transformation, the CNS can effectively decompose the environment into separate internal models appropriate for the separate transformations.

We found clear support for the composition hypothesis. Movement performance in the combined transformation was superior if subjects had previously learned the separate transformations. In particular, target errors were smaller under the combined transformation after exposure to the separate rotational and viscous 
transformations. However, transfer of learning to the combined transformation was not total. Even if two internal models for the separate transformations were already perfectly learned in two anatomically distinct sites in the brain, it is not at all trivial to find the cascade of these two that can resolve the newly given composition task and to establish functional neural connections between the two. Thus, total transfer of learning should not necessarily be expected.

We also found partial support for the decomposition hypothesis. We observed that performance under the rotational transformation was clearly facilitated by previous learning of the combined transformation. Both target errors and path distances were reduced when subjects had previously been exposed to the combined transformation. However, we did not observe a significant facilitation of performance under the viscous transformation attributable to previous learning of the combined transformation. Thus, although subjects appeared to be able to learn and then recall the visuomotor rotation component of the combined transformation, they were not able to learn and/or recall the dynamic component of the same transformation.

It is not clear to us why learning of the combined transformation should only have transferred to the visuomotor transformation. However, one possible explanation is that, when learning the combined transformation, the subject may have adapted primarily to the visuomotor rotation because of the large target errors initially caused by this transformation. Note that when subjects experienced the separate rotational and viscous transformations before learning the combined transformation, target errors were much larger under the rotational transformation. It stands to reason, therefore, that the best way to reduce target errors under the combined transformation would be to focus on learning the visuomotor component. The subjects in our experiment experienced 50 sets of 10 trials under the combined transformation for a total of 500 movements. By the end of this period, errors levels flattened out (Fig. 2) and approached baseline levels (Fig. 3). Thus, it does not seem likely that further training under the combined transformaton would have led to decreased errors under the subsequent viscous transformation.

There are two ways in which subjects might acquire internal models of components of a combined transformation. One possibility is that internal models of the separate transformations (e.g., visuomotor and viscous) are acquired simultaneously during adaptation to the combined transformation. Ghahramani and Wolpert (1997) have recently proposed such a mechanism. These authors argued that a complex visuomotor task can be divided into simpler subtasks, each learned by a separate module in the brain. Another possibility is that the CNS learns a single internal model of the combined transformation and only later decomposes it into its component parts when required. The present results do not enable us to distinguish between these two alternatives, and further modeling efforts will be required to assess their relative advantages.

Overall, the results of this study are consistent with the general hypothesis that the CNS maintains multiple internal models of different environments or sensorimotor transformations. First, the lack of interference effects between the visuomotor and dynamic transformations (i.e., the absence of order effects) suggests that the CNS learned and maintained distinct internal models for these two transformations. If the CNS used a single or global internal model for both the visuomotor and dynamic transformation, learning of one transformation should interfere with subsequent learning of the other. Further support for this view comes from the finding that subjects could successfully inte- grate previously acquired knowledge of the two separate transformations when faced with a novel, combined transformation.

Support for the multiple internal models hypothesis has recently been provided by imaging studies. Shadmehr and Holcomb (1997) have shown that consolidation of learned internal models in memory involves changes in ipsilateral anterior cerebellar cortex. Imamizu et al. (1998) demonstrated that neural activity can be observed in different parts of the cerebellum corresponding to different kinematic transformations after learning. The authors suggested that the different regions of activation correspond to distinct internal models for the different kinematic transformations. The present results suggest that the notion of multiple internal models can be extended to different classes of transformations, namely, dynamic and kinematic transformations.

\section{REFERENCES}

Brashers-Krug T, Shadmehr R, Bizzi E (1996) Consolidation in human motor memory. Nature 382:252-255.

Conditt MA, Gandolfo F, Mussa-Ivaldi FA (1997) The motor system does not learn the dynamics of the arm by rote memorization of past experience. J Neurophysiol 78:554-560.

Flanagan JR, Rao AK (1995) Trajectory adaptation to a nonlinear visuomotor transformation: evidence for motion planning in visually perceived space. J Neurophysiol 74:2174-2178.

Flanagan JR, Wing AM (1997) The role of internal models in motor planning and control: evidence from grip force adjustments during movements of hand-held loads. J Neurosci 17:1519-1528.

Ghahramani Z, Wolpert DM (1997) Modular decomposition in visuomotor learning. Nature 386:392-395.

Gomi H, Kawato M (1996) Equilibrium-point control hypothesis examined by measured arm-stiffness during multi-joint movement. Science 272:117-120.

Imamizu H, Shimojo S (1995) The locus of visual-motor learning at the task or manipulator level: implications from intermanual transfer. $\mathbf{J}$ Exp Psychol Hum Percept Perform 21:719-733.

Imamizu H, Uno Y, Kawato M (1995) Internal representations of the motor apparatus: implications from generalization in visuomotor learning. J Exp Psychol Hum Percept Perform 21:1174-1198.

Imamizu H, Miyauchi S, Tamada T, Sasaki Y, Takino R, Yoshioka T, Pütz B, Kawato M (1998) Multiple representations for visuomotor learning in the cerebellum: a functional MRI study. NeuroImage 7:S819.

Kalaska JF, Cohen AD, Hyde ML, Prud'homme M (1989) A comparison of movement direction-related versus load direction-related activity in primate motor cortex, using a two-dimensional reaching task. J Neurosci 9:2080-2102.

Kawato M, Wolpert DM (1998) Internal models for motor control. In: Sensory guidance of movement (Glickstein M, ed), pp 291-307. Chichester, UK: Wiley.

Lacquaniti F, Guigon E, Bianchi L, Ferraina S, Caminiti R (1995) Representing spatial information for limb movement: role of area 5 in the monkey. Cereb Cortex 5:391-409.

MacGonigle BO, Flook JP (1978) Long-term retention of single and multistate prismatic adaptation by humans. Nature 272:364-366.

Shadmehr R, Brashers-Krug T (1997) Functional stages in the formation of human long-term motor memory. J Neurosci 17:409-419.

Shadmehr R, Holcomb HH (1997) Neural correlates of motor memory consolidation. Science 277:821-825.

Shadmehr R, Mussa-Ivaldi FA (1994) Adaptive representation of dynamics during learning of a motor task. J Neurosci 14:3208-3224.

Sheidt RA, Conditt MA, Reinkensmeyer DJ, Mussa-Ivaldi FA (1997) Motor adaptation persists in the absence of kinematic errors. Soc Neurosci Abstr 23:85.4.

Welch RB (1986) Adaptation to space perception. In: Handbook of perception and human performance (Boff KR, Kaufman L, Thomas JP, eds), pp 24-1-24-45. New York: Wiley.

Welch RB, Bridgeman B, Anand S, Browman K (1993) Alternating prism exposure causes dual adaptation and generalization to a novel displacement. Percept Psychophys 54:195-204.

Wolpert DM, Kawato M (1998) Multiple paired forward and inverse models for motor control. Neural Networks 11:1317-1329.

Wolpert DM, Miall RC, Kawato M (1998) Internal models in the cerebellum. Trends Cognit Sci 2:338-347. 